\title{
Majority Vote method for preferences detection: Application for Social Networks
}

\author{
Mounir Dhibi ${ }^{1}$, Jitendra Pandey ${ }^{1}$, Amine Al Kilani ${ }^{2}$ \\ ${ }^{1}$ MEC, KOM P.B. No. 79, Rusayl, Postal Code: 124, Muscat, Oman, mdhibi@mec.edu.om, \\ jitendra@mec.edu.om \\ 2 UPMI Gafsa , ISSAT Gafsa 2100, Tunisia, aminekilani@gmail.com
}

\begin{abstract}
Most of recommender systems rely on the users' preferences to recommend items. With the increase of data and the number of users on the internet, the task of recommender system becomes more and more sophisticated. The fusion of users' preferences is a solution to facilitate that task. The resolution of this issue, helps in decreasing the run time of a recommender system. This research focuses on the issue of preference fusion in the context of social network. The aim of this research is to determine the collective preferences of users belonging to a given community based on their individual preferences. In particular, researchers are interested here in the representation of individual preferences, taking into account the uncertainty and imprecision phenomenon. In this research two methods are proposed: majority vote method and the Dempster Shafer theory of evidence. Experiments on generated datasets would highlight the interest of the proposed solution.
\end{abstract}

Key words: Fusion; classification; combination; Dempster Shafer theory; detection of preferences; social network.

\section{INTRODUCTION}

Any decision making is based on the preferences that constitute the crucial component in many fields in order to choose the best and most appropriate decisions. Any scientist has to build the most convenient and relevant model to his situation, hence preference modeling is the more important step in a great variety of fields such as mathematical programming [1], biology [2], political science [3], economy [4], [5], computer science [6], databases [7], [8], artificial intelligence [9], ... etc.

Within the field of artificial intelligence, the area of multiagent preference is witnessing a growing interest. Simply stated, in some system, each agent expresses their preferences towards a possible set of decisions. These individuals' preferences will be aggregate to a collective preference of the group in a centralized system. In [11], [12] the authors present definitions, concepts and tools which can be used to make collective decisions in multi agent system.

In social networks, which are similar to a multi-agent system, modeling the preferences is very important especially in the development and implementation of recommendation system (also known "recommender system") which is under incessant development. It's a mechanism that recommends or helps users find some needs like:

- Products as the Amazon system and Alibaba system. This kind of system helps in searching some products and recommends other products to the users.

- Webpages, as Google: by using Google, some pages are recommended based on the search history of the user, the visited sites ...etc.

- Music: where music is recommended according to the sentiments of the user extracted from social networks [16].

- Friends, in social network like Facebook and twitter where the system recommends some users to be friend with or help in finding friends based on the individuals interests.

The context of "modeling the preferences" of users in social networks can help in refining and ordering the result of a web search based on the preferences or interests of a user and his friends [13]. For instance Google provides different results of a search for many users using the same words [10].

Nowadays, sociologists declare that the causes of social phenomena need to be initiated by studying groups instead of individuals [15]. Thereby comes this idea, instead of studying the preferences of each user authors will model and merge the preferences of the communities. A community in a social network is a set of users who interact with each other more frequently and often share the same preferences and interests.

To fuse the preferences, many techniques of data fusion are used in literature for instance Support Vector Machine (SVM) [16], Hidden Markov Models (HMM) [17], possibility theory [7] Artificial Neural Network (ANN) 
[15], majority vote method, Bayesian approach [20], belief functions [27]...etc.

In this article, the modeling of the user's preferences will be studied and a graphic representation for those preferences will be used. For this reason the graph representation; and then two techniques of data fusion to merge the users' preferences in a community will be used. Major contribution in this research consists in presenting the preferences of all users in a community, instead of studying the preferences of each user. This method allows to minimize the run time of a recommender system, and comparing the belief function and the majority vote method.

\section{STATE OF THE ART}

\subsection{Social Networks}

Many definitions of social networks have been proposed in literature. In [22] the author defines social network as an approaches of interactions or relationships, where the nodes consist of actors, and the edges consist of the relationships or interactions between these actors. According to [23], social network site (SNS) provides a new way of communication by the use of computers as a means to increase the speed of forming groups and to expand the group area and influence.

Nowadays, an enormous quantity of data is generated while users, forming networks, are using the social platforms to play games, to do their job, or to socialize... etc. The huge number of users and connections and the enormous quantity of data conduct researchers to study social networks for many aims. Many tasks may be studied:

- Network Modeling: can be used to study different properties of a given network e.g the diffusion of information within the network. Due to the huge number of users, Network modeling becomes a real challenge. A fair amount of work has been done to model networks [24]

- Influence Modeling: is very important to figure out the process of influence. In [9] authors focus on the propagation of information and the identification of subset for nodes that influence more than the others. Among goals to study and their influence.

- To determine customers who influence more their friends, it's known byword-of-mouth marketing

- To study the spread of a new idea

- Privacy: is a substantial and paramount topic some social networks (e.g Facebook, Twitter...etc.) are often suspected about user privacy and even though they reveal some parts of their datasets to be studied anonymously just a description of their interactions or relationships.

- Community detection: is one of the fundamental areas of study, it consists of finding communities in a social network. Till now, there's no specific or exact definition of communities in social network. Authors in 5 define community as follows: "They are usually considered as groups of nodes, in which intra-group connections are much denser than those inter-group ones", they cannot be quantified by numbers. Other terms used for communities such as: clusters, partition, modules or cohesive subgroups. [19], [21].

Lately, researchers have focused more on algorithms or methods to ease the detection of communities in networks. Over the last few years, a huge amount of surveys have been consecrated to study the methods or the algorithms aiming to detect communities [27]. As many approaches have been proposed, the actual research is going to retain those which have mostly attracted the interest of researchers. Community detection methods can be classified into five classes or categories:

- Graph partitioning

- Hierarchical clustering

- Optimization methods

- Based model algorithms

- Hybrid Approaches

Classification and recommendation are common tasks in social networks. A successful social network provides recommendation for every user like friendship, pages, groups etc. For instance, a common feature on the majority of social network is the friendship recommendation, the SNS recommends to the user a number of friends that they might know like old ones, classmates, old neighbors. And due to the huge number of users in a given social network, it's so hard for a user to know who is connected to the site; recommender system is the easiest way to bring other users who may have the same interests or preferences with them. A large variety of recommendation techniques exists. Through the research works, various attempts to classify approaches or techniques were performed. The classification of these methods depends particularly on the type of data and the learning method used by the recommendation system, where the main types of the $R S \mathrm{~s}$ are:

- Content-Based Filtering

- Collaborative Filtering

- Hybrid recommendation

\subsection{Preferences modeling}

Most of the work done in preferences modeling are started up with a set $X$ of "objects" to be compared or to be evaluated. According to the context, this set can be finite 
or infinite. Let us consider a couple $(x, y)$ of objects. To compare the two objects " $a$ " and " $b$ " hence 3 situations are existing:

- The preference of one of the two objects to the other.

- Indifference between the two objects.

- In-comparability between the two objects.

These 3 situations can be noted as follows:

- $a<b$ resp $a>b$ if $a$ is preferred to $b$ (resp $\mathrm{b}$ is prefred to $a$ )

- $a=b$ if $a$ and $b$ are indifferent ( $a$ and $b$ with the same degree will be given preference)

- $a \neq b$ if $a$ and $b$ are comparable (car can't be comparable to food)

These 3 situations are used in the most works dealing with the preferences modeling [93]. Given $a$ set $A$ of objects,

- All the couples $(a, b)$ such as $a \prec b$ establish the preference relation, noted $P$; it is natural to consider that this relation is asymmetric: i.e $P a$ is not possible, and if $a P b$, then $b P a$ is excluded.

- All the couples $(a, b)$ such as $a=b$ establish the relation of indifference, noted $I$; it is natural to consider that this relation is reflexive i.e $a I a$ is always possible and symmetric i.e. if $a I b$, then $b I a$

- All the couples $(a, b)$ such as $a \neq b$ establish the relation of in-comparability, noted $R$; it is natural to consider that this relation is deliberate i.e $a R a$ is not possible and symmetric i.e. if $a R b$, then $b R a$

Table 1: Graphic representation

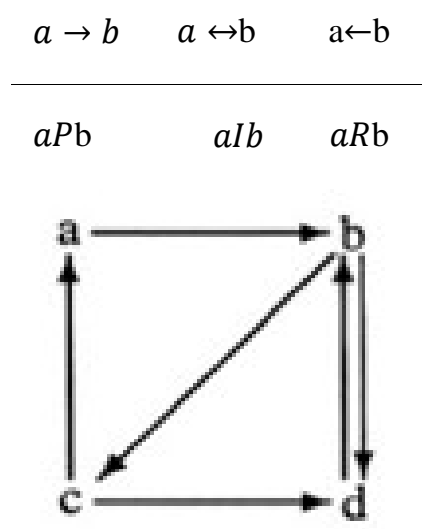

Figure 1: Graphical representation.

It is considered that the three relations $(P, I, R)$ form the preferences structure on $A$ only if they have the properties indicated above, and for all couple of elements $a$ and $b$ there is only one of the following possibilities:

- $a P b$

- $b P a$

- $a I b$
- $a R b$

In this work, authors have adopted the following representation to represent the preferences structure as mentioned in Table 1.

It can be convenient to represent a preference structure by a matrix as in table 2. This representation may facilitate the task of reading the preferences between objects in case of a huge number of objects. Also called adjacency matrix. In mathematics, an adjacency matrix, for a finite graph with $n$ nodes, is a $n \times n$ matrix where the non-diagonal element $a_{i j}$ represents the number of edges linking the node $i$ to the node $j$. the diagonal element $a_{i j}$ is the number of loops in that node.

In graph theory, the adjacency matrix contains only "0" and "1" , if $a_{i j}=0$ means that there is no link between the node " $i$ " and the node " $j$ " and if $a_{i j}=1$ means there is a link between $i$ and $j$.

Example: let $a$ be set of objects $A=\{a, b, c, d\}$ authors have assumed the following preference structure:

- $P=\{(a, b),(b, c),(c, a),(c, d),(d, b)\}$

- $I=\{(b, d)\}$

- $R=\{(a, d)\}$

The table 2 shows graphical representation and the matrix description of the example.

Table 2: Matrix representation

\begin{tabular}{l|l|l|l|l}
\hline & $\mathrm{a}$ & $\mathrm{b}$ & $\mathrm{c}$ & $\mathrm{d}$ \\
\hline $\mathrm{a}$ & 0 & 1 & 0 & 0 \\
$\mathrm{~b}$ & 0 & 0 & 1 & 1 \\
$\mathrm{c}$ & 1 & 0 & 0 & 1 \\
$\mathrm{~d}$ & 0 & 1 & 0 & 0 \\
\hline
\end{tabular}

\subsection{Data fusion and proposed methods}

\subsubsection{Definition:}

Data fusion is represented by a set of scientific methods to create or refine indicators by fusing data from heterogeneous sources or sensors. These sensors or sources may be: radar, therma, acoustic, laser, optical, sonar analysis, or chemical detection devices etc. The field of data fusion has multiple uses [17]. It is generally used for the indicators for which there is no estimation method or making reliable direct decision and / or easy to implement. Data fusion is a specially information integration problem [17]. Therefore, integrating data from multiple sensors of different types provides a better result because the strengths of one type can compensate for the 
weaknesses of another type. In simple words data or information fusion consists in combining data from many sources to ameliorate making decisions. The data fusion process consists of 4 steps [26]. Firstly, the modeling task was defined by how the information is represented by experts. The second step describes the estimation information: it depends on the modeling where authors give measure reliability to some sources. The most important step is to determine how data are combined, this is the third step. Finally, we use information issue from combination step to make an appropriate decision. Conceptually, data fusion is a class of problems appropriate for many applications like: Environment, military, medical, economics etc. 2.3.2. Notations and positioning:

- $S=\left\{s_{1}, s_{2}, \ldots s_{n}\right\}$ denotes the set of sources in a system where $n$ is the number of all sources. Each source is represented by $S_{j}$

- $D=\left\{d_{1}, d_{2}, \ldots d_{n}\right\}$ denotes the set of possible decision where $m$ is the number of all possible decisions. Each decision is represented by $d_{i}$

- $M=$ Matrix containing the set of observation of each source $s_{j}$ on every decision $d_{i}$

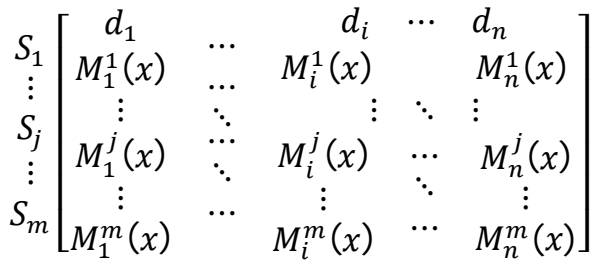

Where $M_{i}^{j}(x)$ represents the observation of the $j^{t h}$ source on the $i^{\text {th }}$ decision.

\subsubsection{Proposed methods:}

Among the methods used for data fusion, Vote method is the simplest one to be implemented. And it has only three steps because there's no estimation step [26]. In this research, $N_{c}$ decisions will be made as decision for each pair of object is required.

For this method, the decisions $d_{i}$ are exclusives.

- Modeling step:

$$
M_{i}^{j}(x)=\left\{\begin{array}{c}
1 \text { if } U^{j}(x)=i \\
0 \text { otherwise }
\end{array}\right.
$$

Where $U^{j}(x)=1$ means that the user chooses situation $i$ for the pair $j$

- Combination step: The combination of the sources is given by:

$$
\begin{aligned}
& M_{i+1}^{j}(x) \\
& =\sum_{i=1}^{n} M_{i}^{j}(x) \quad \text { for all } j
\end{aligned}
$$

Decision step: In this step each pair is to be decided, i.e. the situation where it has the maximum between fourth ones has been taken. And if result is two maximums, it means that decision for that pair can be taken.

$$
E(x)=\left\{\begin{array}{c}
i \text { if } \max M_{i+1}^{j}(x)>\frac{u}{2} \text { for each combination } \\
0 \text { otherwise }
\end{array}\right.
$$

The rule of majority vote consists in choosing the decision taken by the maximum of users. However this rule conducts sometimes to a non-decision i.e. in case where the number of users $u$ is a pair number and $u / 2$ of users decide $S_{1}$ and the others $u / 2$ sources decide $S_{2}$, a nondecision is faced here because which one to make between the two situations can't be decided. So decision is added $d_{n+1}$ which represents the uncertainty. So, the best combination is applied when the outputs are independent and an odd number of sources is achieved. The majority vote method has many advantages, for example, it is easy to use or implement and it doesn't need a prior knowledge.

Algorithm 1: Fusion of $n$ users and $m$ objects by Majority Voting Method.

Input : $n, m$ : integer

Output: Graph for the $m$ objects

Var :

$N c$, nocol, Long, $Q$ : integer Nc, nocol, Long, Q: integer

$V f$, $R f$, Res : vector Mf : matrix Max: real begin

$\mathrm{nc}=(\mathrm{m} \times(\mathrm{m}-1)) / 2 \mathrm{nocol}=\mathrm{nc} \times 4$

Generation of the matrix(nuser, nocol)

$\mathrm{Vf} \leftarrow$ Sum of each column

$\mathrm{Vf} \leftarrow \mathrm{Vf} /$ nuser

Mf $\leftarrow$ matrix(vf, nrow=nc, byrow) $\%$ Transform of vf into matrix with number of possible combination and 4 columns for the 4 situations;

for $i \leftarrow 1$ to $n c$ do

$\operatorname{Max} \leftarrow \max (\operatorname{Mf}[i]$,$) ;$

Long $\leftarrow$ number of duplicated Max;

if $($ long $=1)$ then

$\mathrm{Q} \leftarrow$ index of the Max;

$\operatorname{Res}[\mathrm{i}] \leftarrow \mathrm{Q}$;

end else

$\underset{\text { end }}{\operatorname{Res}[\mathrm{i}] \leftarrow 0 \% \text { no decision; }}$

end

Creation of adjacency Matrix;

Plot of the graph; 
The algorithm 1 explains steps of the preference fusion for $\mathrm{n}$ users with m objects.

Case study: To better explain the process of this method, authors are going to present the approach with 3 persons and 4 objects. So three of them express their preferences toward 4 objects by pair wise. So figure 2 illustrate the approach; it is assumed that there are three users who have expressed their preferences: a single arrow

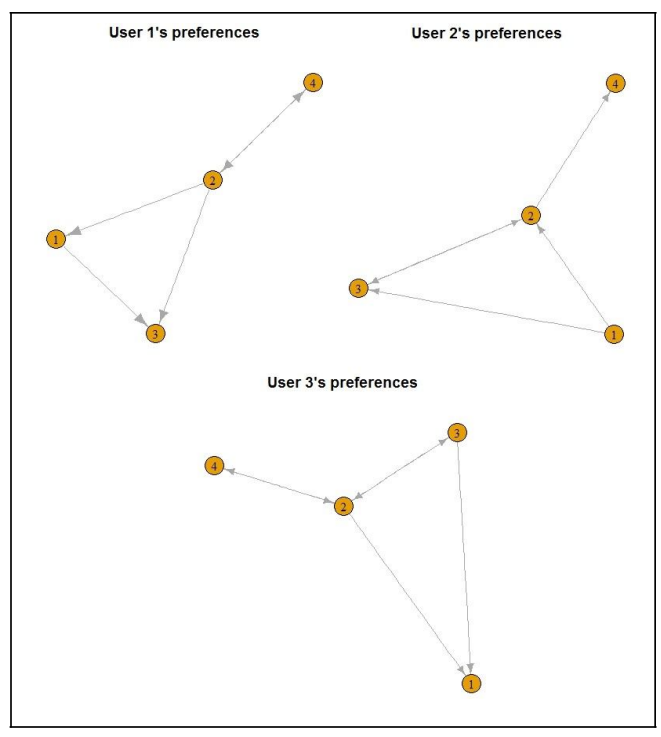

Figure. 2: Preferences of 3 users.

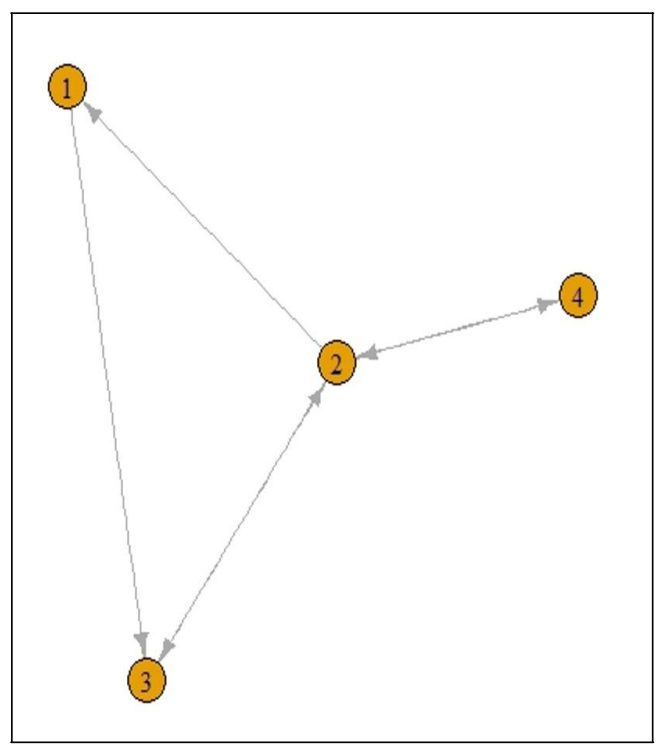

Figure 3: The result of fusion for 4 objects.

indicates preferably, a double one the indifference and incomparability without arrow.

The result of the fusion process was indicated in figure 3 . Results thus shows the object' 2 being preferred to the object' 1 by two of the three, preference of the group will remain Object' 2 preferred to the object' 1 . The indifference between objects' 2 and ' 4 for the first person and the third person remains indifferent after fusion process and so forth.

Preferences fusion based on the Dempster Shafer Theory: The Dempster-Shafer theory of evidence was first proposed by Arthur.P Dempster in 1967 and Glenn Shafer in 1976 and was also known as the theory of belief functions [28]. This theory is a generalization of the Bayesian one's of subjective probabilities. The major difference between DST and Bayesian fusion is that the beliefs are assigned to sets in the first one and to elements in the latter one. The frame of discernment $\Omega=$ $\left\{\Omega_{1}, \Omega_{2}, \ldots, \Omega_{n}\right\}$ is the base of DST, it's an exhaustive set of mutually exclusive alternatives or decisions of a given problem. And the set noted $2^{\Omega}$ consists of all possible subsets formed by decisions and union of decisions from $\Omega$ and for this reason the DST allows us to model the information's imprecision. It also contains the empty set which represents the conflict.

\section{- Modeling}

A belief distribution mass (bba: basic belief assignment) $m$ represents the belief on subsets of the frame of discernment and it's defined:

$$
\begin{gathered}
m: 2^{\theta} \rightarrow[0,1] \\
\sum_{A \subseteq \theta} m(A)=1
\end{gathered}
$$

Where $A$ is $a$ subset of $\Omega$. For the mass of empty set there are 2 possibilities:

- $m(\emptyset)=0$ in this case is is assumed that all decisions are exhaustive.

- $m(\emptyset)>0$ in this case a decision may be accepted which is not included.

The mass function represents one of many functions used in this step, among them plausibility function, belief function, communality function etc. can be cited [27], [29].

\section{- Estimation}

In this step, we can affect a specific quantity of belief on a special set, for example it can be estimated that a subset has an important belief more than the other subsets.

\section{- Combination}

The combination is an important number of contributions were done to propose new rules. In [27], [29] authors present one of many surveys about combination rules in the DST. And among those rules Conjunctive rule, Yager rule, Inagaki rule etc. is selected. 
Authors focus here on the conjunctive rule or the Dempster rule. This rule allows combining two mass functions or more to one function. For two functions the rule is written as below:

$$
\begin{gathered}
m(A)=m_{1} \oplus \cdots \oplus m_{m}(A)= \\
\sum_{B_{1} \cap \cdots \cap B_{m}=A} \prod_{j=1}^{m} m_{j}\left(B_{j}\right)(5)
\end{gathered}
$$

For all $A \in 2^{\theta}$ and for the m mass function is written:

$$
m(A)=m_{1} \oplus m_{2}(A)=\sum_{B \cap C} m_{i}(B) m_{2}(C)
$$

This rule is used when authors dispose of sources inducing masses are known and telling the truth and independents, which means that they are assumed to provide a specific and non-overlapping evidences.

\section{- Decision}

This is the last step; different criteria to allow making the decision are presented: maximum of plausibility, maximum of belief, or of pignistic probability. The latter one was introduced by Smets in [30]. The algorithm 2 represents all steps of the DST.

$$
\operatorname{bet} P(X)=\sum_{Y \in 2^{\theta}, Y \neq \phi} \frac{|X \cap Y|}{|Y|} \frac{m(Y)}{1-m(\phi)}
$$

Besides to create the adjacency matrix, matrix match is created containing all the possible combination of pairs and two null matrix Algorithm 3. One for the decisions and other for possible non decisions only for the case of majority vote method and DST only one matrix is needed.

\section{RESULT AND ANALYSIS}

Due to the lack of a suitable datasets that can be used to test our work, an artificial dataset for each method is created.

\subsection{Datasets}

As aforementioned in section 2.2, four attitudes exist to compare two objects " $a$ " and " $b$ ".

- $a>b: a$ preferred to $b$

- $a<b: b$ preferred to $a$

- $a=b: a$ and $b$ are indifferent

- $a b: a$ and $b$ are incomparable

So a user $u(u \in 1, \ldots$ etc., $U)$, expresses his preferences by comparing a pair of objects from $(1, \ldots e t c ., n)$. The number of possible combination is determined as follows:

$$
N_{c}=\frac{[n \times(n-1)]}{2}
$$

Algorithm 2: Different steps of DST of $n$ users and $m$ objects by Majority Voting Method.

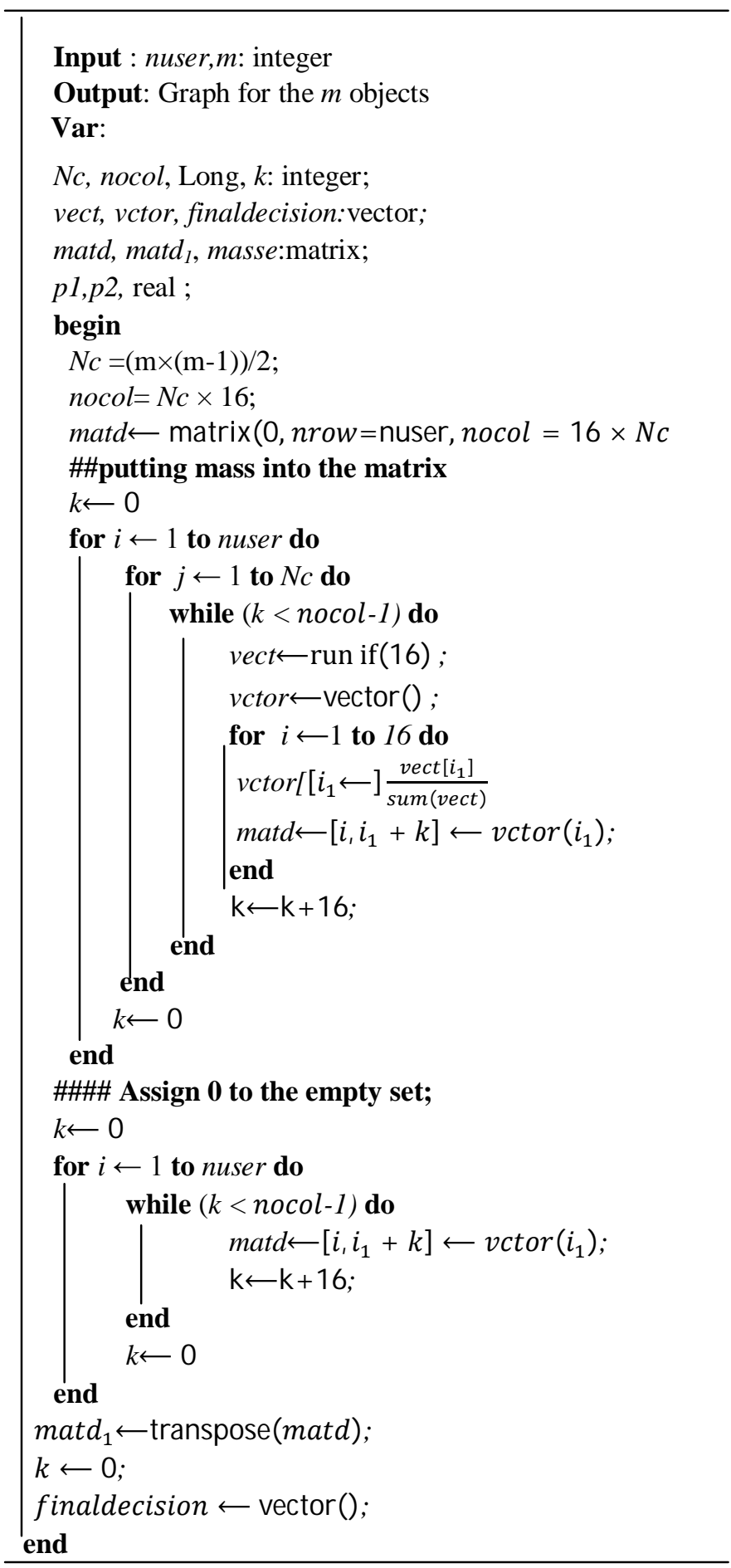


Algorithm 3: Creation of adjacency matrix
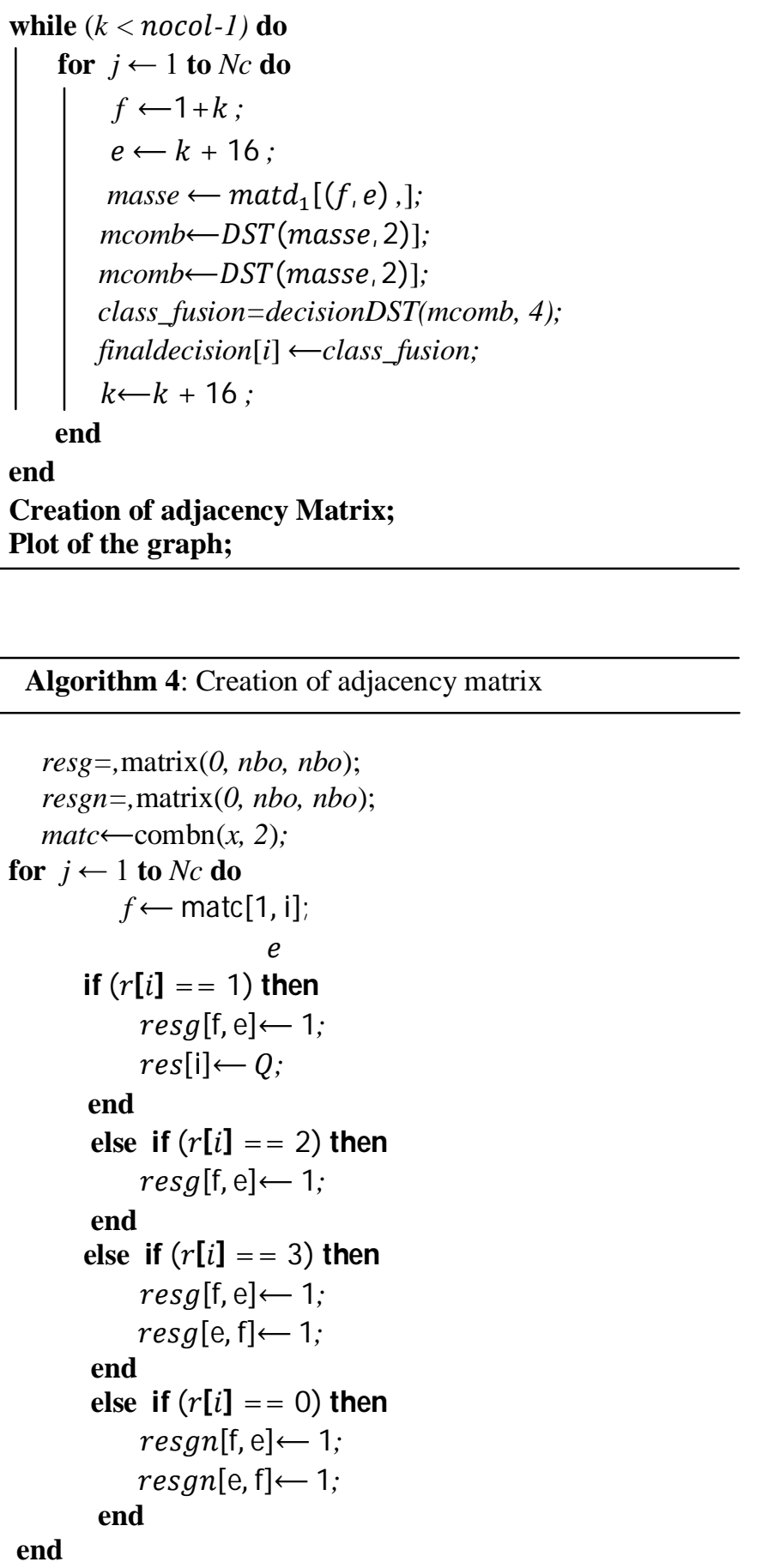


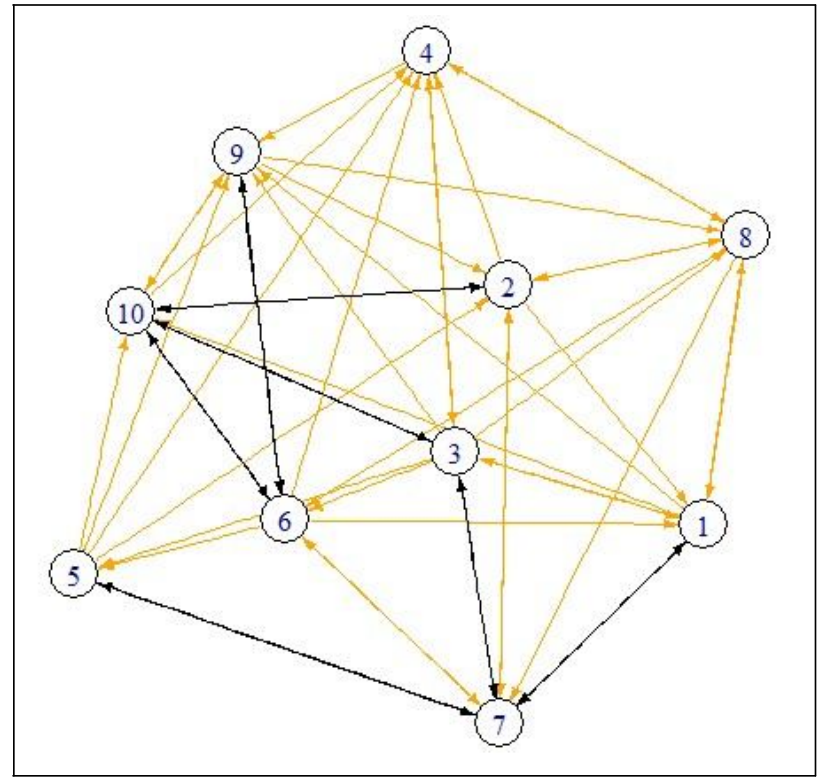

Figure 4: MVM for 10 objects.

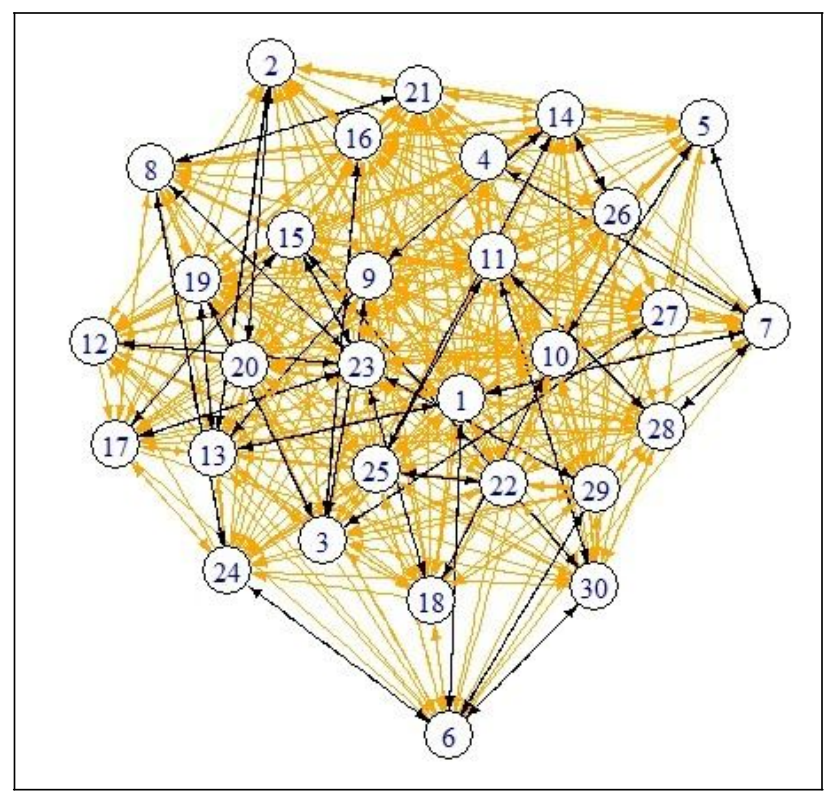

Figure 5: MVM for 30 objects.

\subsection{Experimental results}

All the experiments were implemented using $R$ software due to his powerful scripting, his free cost and its ease of use. These experiments can be run on a windows 7 based PC with Intel Core Quad processor having a speed of 2.67 $\mathrm{GHz}$ and $8 \mathrm{~GB}$ of Ram

Majority Vote results: Experiment was started by generating 5000 users' preferences towards 10, 30, 100 objects. So there are respectively 45, 435, 4950 combina-

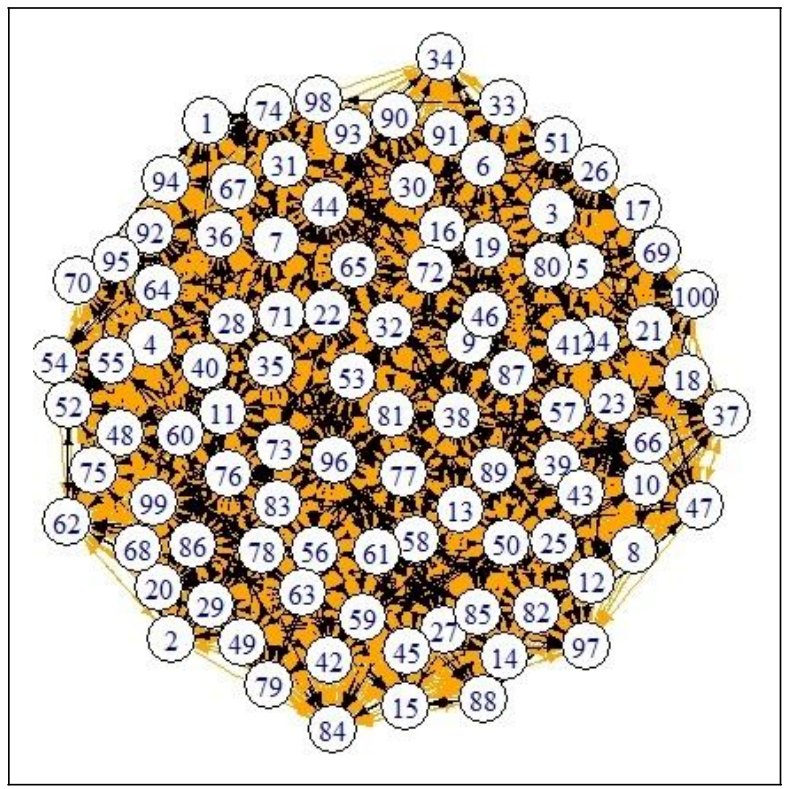

Figure 6: MVM for 100 objects.

tions on which each user decides his preference for each combination (preferred to, indifferent or incomparable). The result of the fusion is represented in figure 7 . The black arrow means that for that pair there is no decision and for the others there is decision either preferred to, or indifferent, or incomparable.

Big number of experiments was done by increasing the number of users and checked the number of non-decision was checked. It was noticed that the number of nondecision decreases, when the number of users increases, this will be proved later mathematically. Figure 7 justifies our remark. For 10 objects fusion of preferences was made for 20,100 , and 300 users and it was found: -8 cases of non-decision for 20 persons

- 6 cases of non-decision for 100 persons - 3 cases of $\mathrm{c}$ for 300 persons

- Just one case of non-decision for 500 persons To prove our remark mathematically, a simple example is given using only two objects $A$ and $B$ and number of users are successively increased.

For 2 users, the possible combination will be: As it can be seen there is only one non-decision, so the probability to have it is equal to $1 / 3$. Now let's see the probability of non-decision for 6 users. Here the probability to have a non-decision is $1 / 7$. So for $\mathrm{n}$ users the probability to face a non-decision is $\frac{1}{(n+1)}$ and this can explain the remark mentioned above.

Belief Function results: Also for this method a 5000 users' preferences are generated toward 10,30, and 100 


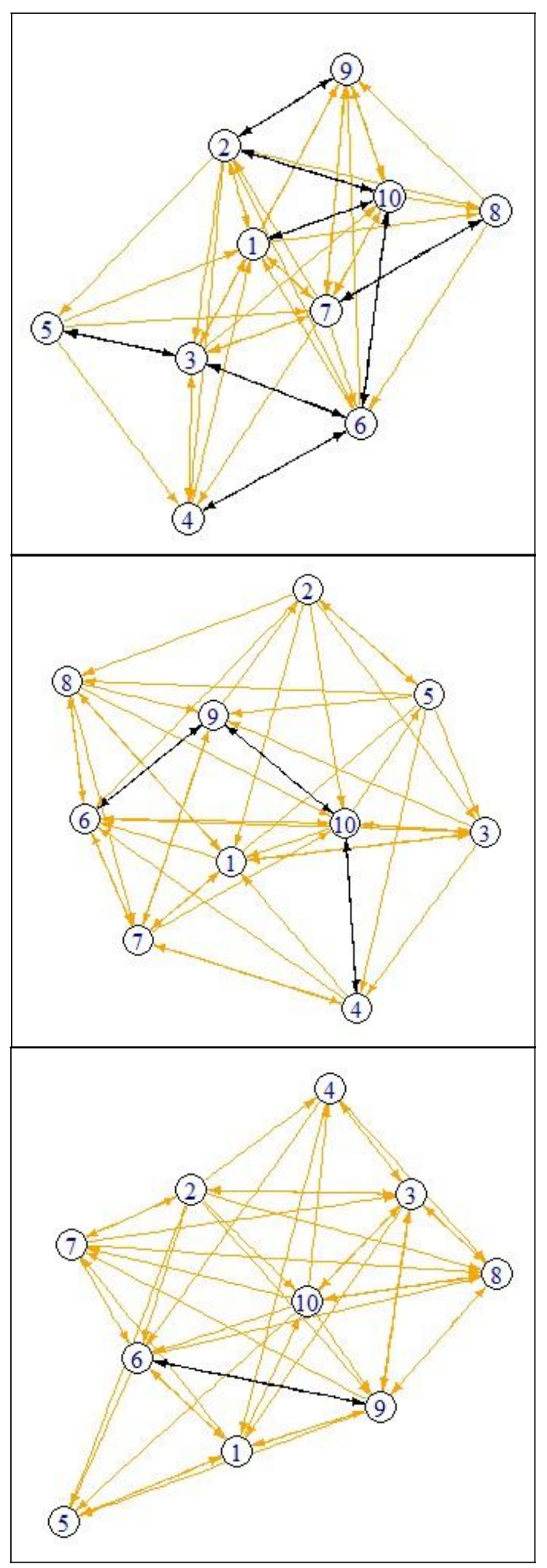

Figure 7: Non decision cases.

objects. Figures 8, 9 and 10 show the results of DST fusion. For the fusion of preferences of users for 10 .
Table 4: Vote of 2 users towards two objects $A$ and $B$

\begin{tabular}{cc} 
A & B \\
\hline 2 & 0 \\
0 & 2 \\
1 & 1 \\
\hline
\end{tabular}

Table 5: Vote of 6 users towards two objects A and B

\begin{tabular}{cc}
\hline $\mathrm{A}$ & $\mathrm{B}$ \\
\hline 6 & 0 \\
0 & 6 \\
5 & 1 \\
1 & 5 \\
4 & 2 \\
2 & 4 \\
3 & 3 \\
\hline
\end{tabular}

objects it's easy to read the results, but to fuse the preferences of users toward a big number of objects the figure becomes more dense. As mentioned the last section solution is to use the adjacency matrix. For example, in the fusion of preferences of 100 objects for the two objects 29 and 30 the result can't be seen but by checking the adjacency matrix it is found that $M[29,30]=1$ and $M[30,29]=1$ so the two objects are indifferent. And for each pair the adjacency matrix is checked to get the information about preferences for that pair. Among the imperfections aspects of information imprecision is found. This method can be used to model on one hand the uncertainty and on the other hand it also allows the modeling of information's imprecision. This method allows to model the ignorance of the user about his preferences, this is not possible in the both method: majority vote and the Bayesian approach So this method can be considered better than the two first methods due to its flexibility to the model of imprecision which is a real issue in the real world especially with humans.

\section{CONCLUSION}

In this work, author's focused on the different tasks in social networks mainly the recommendation. The last one takes into account the user's preferences and interests. One of the RS system is the collaborative filtering one. It's based on the users' behaviors by analyzing and collecting a huge amount of information, activities or 


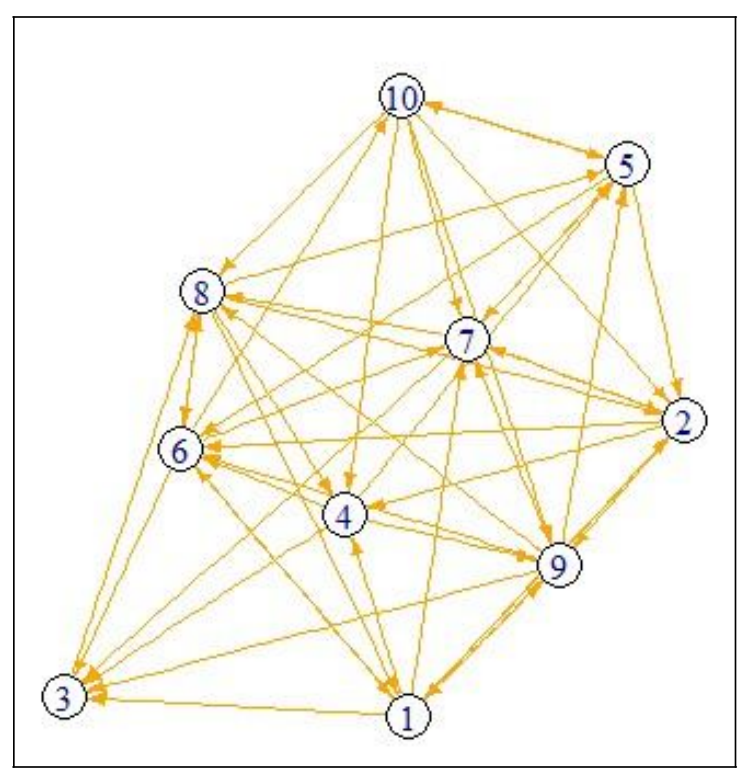

Figure 8: DST for 10 Objects.

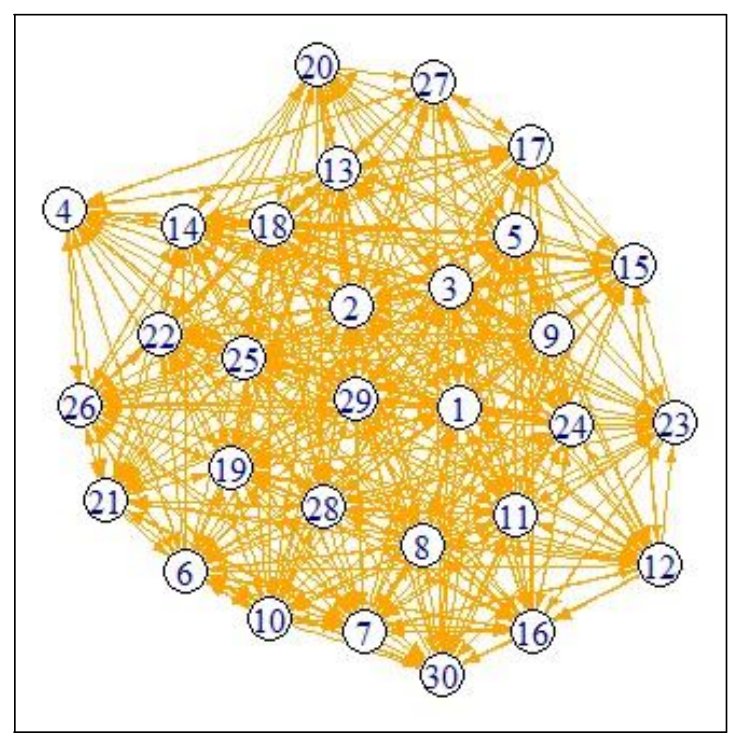

Figure 9: DST for 30 Objects.

preferences and predicting what users will like based on their similarity to other users. Here comes the main idea of this work. Instead of recommending objects to each user, preferences' fusion can be made in a community to recommend those objects to the whole members of that community.

The major hypothesis is that the user can express his/her preferences toward a pair of objects by four attitudes (preferred to, indifferent and incomparable). The preferences of users can be represented in different ways;

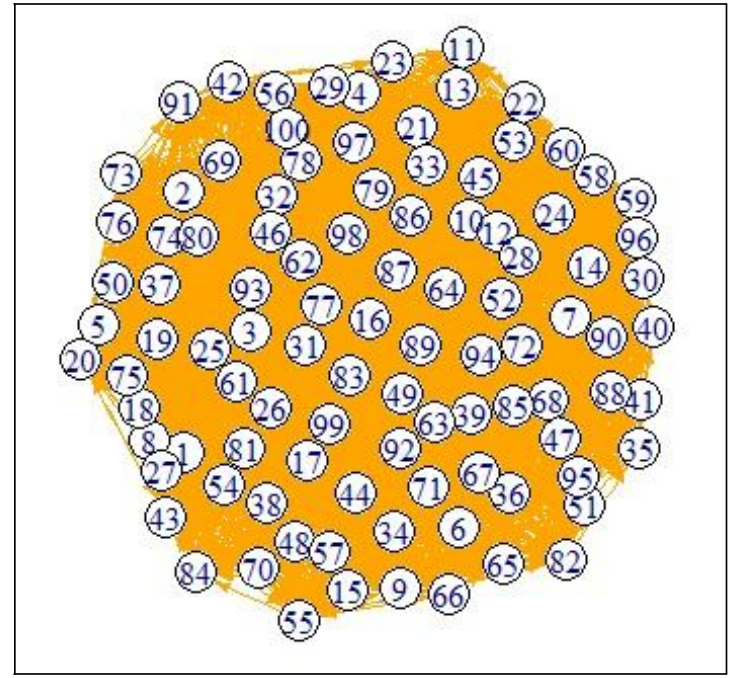

Figure 10: DST for 100 Objects.

matrix representation and graphic representation. The aim of this work has been to study the fusion's techniques and use some of them to fuse the preferences of different users. The major obstacle authors have faced is the lack of datasets that allows us to test our work and to compare it with other works in terms of complexity, run-time, allocated memory, etc. To overcome that obstacle, two datasets were created; each one is dedicated for one of the two methods: majority vote method and the DempsterShafer theory of evidence. To solve the issue of preferences fusion in the context of social network, we have used the two methods mentioned above. All the techniques provide encouraged results, however for the Majority Vote method, A major drawback was noticed which is the possibility of non-decision if it exists two maximum of decision are equals. For each one of these methods, a dataset was generated to test our application. This data contains 5000 users where the preferences of each one is presented in a matrix and number of objects might be varied. Both methods provides results but we recommend the use of DST due to its advantages cited in this paper.

\section{REFERENCES}

1. J. Doyle and M. P. Wellman. Modular utility representation for decision theoretic planning, Proceeding of the First International Conference on Artificial Intelligence Planning Systems, pp. 236-242, 1992.

2. G. Debreu. Theory of value: an axiomatic analysis of economic equilibrium, John Wiley and Sons Inc., New York, 1959. 
3. A. K. Sen. Social choice theory, in K.J. Arrow et M.D. Intriligator (eds), Handbook of Mathematical Economics, Vol. 3, pp. 1073-1181, 1986.

4. M. Lacroix and P. Lavency. Preferences: Putting more knowledge into queries, In Very Large Data Bases (VLDB), pp. 225, 1987.

5. K. Werner. Foundations of preferences in database systems, In Proceedings of the 28th international conference on Very Large Data Bases (VLDB), pp. 311-322, 2002.

6. M. S. Abari and Craig Boutilier. Preference-oriented Social Networks: Group Recommendation and Inference, In RecSys 15: Proceedings of the 9th ACM Conference on Recommender Systems, pp. 35-42, Vienna, Austria.

7. T. Denoeux and M. H. Masson. Evidential reasoning in large partially ordered sets, Annals of Operations Research, V. 195(1), pp. 135-161, 2012. https://doi.org/10.1007/s10479-011-0887-2

8. B. Roy. Preference, indifference, incomparabilite, document, LAMSADE N. 9, Paris, 1980.

9. O. Shafiq, T. N. Jarada, PanagiotisKarampelas, RedaAlhajj, and J. G. Rokne. Integrating Online Social Network Analysis in Personalized Web Search The Influence of Technology, on Social Network Analysis and Mining, pp. 589-613, 2013.

10. A. S. Das, M. Datar, A. Garg and S. Rajaram. Google news personalization: scalable online collaborative filtering, In Proceedings of the 16th international conference on World Wide Web, pp. 271-280, 2007.

https://doi.org/10.1145/1242572.1242610

11. J. K. Lou, F. M. Wang, C. H. Tsai, P. H. Kung, and S. D. Lin. Modeling the diffusion of preferences on social networks, In Proceedings of SIAM international conference on data mining SDM, pp. 1247-1252, 2013.

https://doi.org/10.1137/1.9781611972832.67

12. J. Zou and F. Fekri. On Top-N Recommendation Using Implicit User Preference Propagation over Social Networks, In Proceedings of IEEE International Conference on Communications Social Networking Track (ICC'14), Sydney, Australia, Jun. 2014.

https://doi.org/10.1109/ICC.2014.6883933

13. R. Goyal, P. Dhyani, O. P. Rishi. Like-A unique way to judge user preference in social networking sites, Fifth International Conference on Communication Systems and Network Technologies, pp. 1056-1059, 2015.

https://doi.org/10.1109/CSNT.2015.182

14. R. L. Rosa, D. Z. Rodriguez and G. Bressan. Music Recommendation System Based on User's Sentiments Extracted from Social Networks, IEEE International Conference on Consumer Electronics (ICCE), pp. 359-367, 2015.
https://doi.org/10.1109/ICCE.2015.7066455

15. M. P. Wellman and J. Doyle. Preferential semantics for goals, In Association for the Advancement of Artificial Intelligence, V. 91, pp. 698-703, 1991.

16. F. Rossi, K. B. Venable, and T. Walsh. A short introduction to preferences : between artificial intelligence and social choice, Synthesis Lectures on Artificial Intelligence and Machine Learning, V. 5(4), pp.1-102, 2011.

https://doi.org/10.2200/S00372ED1V01Y201107AIM 014

17. F. Brandt, V. Conitzer, and U. Endriss. Computational social choice, Multi-agent systems, pp. 213-283, 2012.

18. M. E. Newman, M. Girvan, Finding and evaluating community structure in networks, Physical review, V. 69(2), pp. 026113-15, 2004.

19. V. D. Blondel, J. L. Guillaume, R. Lambiotte, E. Lefebvre. Fast unfolding of communities in large networks, Journal of Statistical Mechanics: Theory and Experiment, 2008. https://doi.org/10.1088/1742-5468/2008/10/P10008

20. J. Duch and A. Arenas. Community detection in complex networks using extremal optimization, Physical review, V. 72(2), 027104, 2005.

21. M. Tasgin,A. Herdagdelen, H. Bingol. Community detection in complex networks using genetic algorithms, arXiv preprint arXiv, 0711.0491. 2007.

22. S. Fortunato and M. Barthelemy. Resolution limit in community detection, Proceedings of the National Academy of Sciences, V. 104(1), pp. 36-41, 2007. https://doi.org/10.1073/pnas.0605965104

23. B. Amiri, L. Hossain,J. W. Crawford, and R. T. Wigand. Community detection in complex networks: Multi-objective enhanced firefly algorithm, Knowledge-Based Systems, V. 46, pp. 1$11,2013$. https://doi.org/10.1016/j.knosys.2013.01.004

24. A. Lancichinetti, S. Fortunato and J. Kertesz. Detecting the overlapping and hierarchical community structure in complex networks, New Journal of Physics, V. 11(3), 033015, 2009. https://doi.org/10.1088/1367-2630/11/3/033015

25. O. H. Bray. Information integration for data fusion, Sandia National Laboratories Report, SAND97-0195. 1997

26. I. Bloch. Information combination operators for data fusion: a comparative review with classification, Systems, Man and Cybernetics, Part A: Systems and Humans, IEEE Transactions on, V. 26(1), pp. 52-67, 1996.

27. Y. Zhang, T. Bouadi, A. Martin. Clustering model for uncertain preferences based on belief functions, 20th International Conference on Big Data Analytic 
and Knowledge Discovery, Regensburg, Germany, 10.1007/978-3-319-98539-8-9, 2018.

https://doi.org/10.1214/aoms/1177698950

28. S. Dempster,Upper and lower probabilities induced by a multi-valued mapping, Ann Math Stat, V. 38, pp. 325-339, 1967.

29. F. Karem, M. Dhibi, A. Martin, and M. S. Bouhlel. Credal fusion of classifications for noisy and uncertain data, International Journal of Electrical and Computer Engineering, V. 7(2), pp.1071-1087, 2017.

https://doi.org/10.11591/ijece.v7i2.pp1071-1087

30. P. Smets. Combination of evidence in the transferable belief model, IEEE Transactions on Pattern Analysis and Machine Intelligence, V. 12(5), pp. 447-458, 1990.

https://doi.org/10.1109/34.55104 